NASA TM X-55894

\title{
THE ELECTROMAGNETIC RADIATION ASSOCIATED WITH THE ENERGY DISSIPATED BY COSMIC RAYS IN INTERSTELLAR HYDROGEN
}

V. K. BALASUBRAHMANYAN

E. BOLDT

R. A. R. PALMEIRA

G. SANDRI

JULY 1967 
The Electromagnetic Radiation Associated

With the Energy Dissipated by Cosmic Rays

in Interstellar Hydrogen

V. K. Balasubrahmanyan and E. Boldt

NASA/Goddard Space Flight Center, Greenbelt, Md.

R. A. R. Palmeira

Southwest Center for Advanced Studies, Dallas, Texas

G. Sandri*

Aeronautical Research Associates of Princeton, Inc.

Princeton, N. J.

* Supported by a grant from the/ National Aeronautics and Space Administration, Goddard Space Flight Center, Greenbelt, Maryland. 
The temperature observed $\left(\sim 10^{2}{ }^{\circ} \mathrm{K}\right)$ for the hydrogen atoms of interstellar $\mathrm{Hl}$ clouds is shown to be a consistent measure of the Coulomb interactions of cosmic rays. These interactions could also give rise to a high electron concentration $\left(\sim 10^{-1} \mathrm{~cm}^{-3}\right)$. The expected intensity of $\mathrm{H} \alpha$ emission by the hydrogen atoms excited by cosmic rays is found to be comparable to but less than that measured from the galactic disk. The inner bremsstrahlung $\mathrm{X}$-radiation by electrons ejected from interstellar hydrogen atoms is indicated as a direct measure of the spectrum of the cosmic rays responsible for the ionization. A detailed study of the non-stellar X-rays from $H 1$ clouds is recommended for the determination of the spatial and spectral distribution of the low energy (< $10^{8} \mathrm{e} . \mathrm{v} . /$ nucleon $)$ cosmic rays that propagate throughout the galactic disk. 


\section{Introduction}

Cosmic ray measurements during the recent period of minimum solar modulation indicate that there is an appreciable flux of low energy highly ionizing cosmic ray particles in the interstellar medium (see Fig. 1). However, the modulation (Balasubrahmanyan, Boldt and Palmeira 1967) appears even at solar minimum to be sufficiently strong and complicated to make a precise inference of the interstellar spectrum quite unlikely unless there is the input of some additional relevant information directly from outside the solar system. This probelm is here approached from the outside by considering the Coulomb interactions of these particles in the interstellar medium and examining the possible electromagnetic signatures that could be measured from observations made from within the solar system.

It is important to note that most of the interstellar gas is in $\mathrm{Hl}$ clouds of neutral hydrogen (for a review see Dieter and Goss, 1966). The Doppler broadening of the $21 \mathrm{~cm}$ line of atomic hydrogen indicates a gas temperature of $\sim 10^{2}{ }^{\circ} \mathrm{K}$ for such clouds. The possible relevance of low energy cosmic rays for maintaining this temperature was noted by Hayakawa (1960). Recent work by Field (1962) and Smith (1966) on the cooling mechanisms for these clouds indicates that the characteristic cooling time $\left(\lesssim 4 \times 10^{4}\right.$ years $)$ is much shorter than the meantime $\left(\sim 7 \times 10^{6}\right.$ years $)$ between the 
cloud-cloud collisions described by Kahn (1955) as the events responsible for most of the heating. Hence, a steady-state input such as the heating by cosmic rays now appears compelling, and the problem is here re-examined within the context of now infomation.

\section{Thermal Balance}

The energy dissipated by cosmic ray nuclei $\left(E>E_{0}\right)$ in the interstellar medium via Coulomb interactions with the constituent hydrogen atoms is given per gram-second by:

$$
q\left(E_{o}\right) \equiv 4 \pi\left[\int_{E_{o}}^{\infty}\left(\frac{d E}{d x}\right)\left(\frac{d J}{d E}\right) \frac{d E}{\eta}\right] \quad \frac{e \cdot v \cdot}{g-s e c}
$$

where: $E=$ kinetic energy per nucleon,

$$
\begin{aligned}
\eta= & \text { fraction of cosmic ray nucleons that appear as } \\
& \text { free protons, } \\
\frac{\mathrm{dE}}{\mathrm{d} x} & \mathrm{e} \cdot \mathrm{v} \cdot \operatorname{loss} /\left(\mathrm{g}-\mathrm{cm}^{-2}\right) \text {, for protons traversing atomic hydrogen, } \\
\frac{\mathrm{dJ}}{\mathrm{dE}} \equiv & \frac{\text { interstellar protons }}{\left(\mathrm{cm}^{2}-\mathrm{sec}-\mathrm{sr}-\mathrm{e} \cdot \mathrm{v} \cdot\right)}
\end{aligned}
$$

The Coulomb interaction of cosmic rays with interstellar hydrogen atoms results in

1) the ejection of atomic electrons,

ii) inner bremsstrahlung $x$-rays generated by the ejecting electrons, and

iii) the excitation of atomic levels. 
The ejected electrons slow down by heating the gas, and finally recombine with ions to produce characteristic hydrogen emission lines. The heated hydrogen atoms cool by collisional excitation of infra-red emitting levels of other atomic species, especially oxygen (Smith, 1966).

Under steady-state conditions, the conservation of energy appears as:

$$
\begin{aligned}
\left(\begin{array}{l}
\text { Cosmic Ray } \\
\text { Heating }
\end{array}\right) & =\left(\begin{array}{l}
\text { Inner } \\
\text { Bremsstrahlung } \\
\text { X-Radiation }
\end{array}\right)+\left(\begin{array}{l}
\text { Hydrogen } \\
\text { Line } \\
\text { Emission }
\end{array}\right)+\left(\begin{array}{l}
\text { Atomic } \\
\text { Cooling }
\end{array}\right)+\left(\begin{array}{l}
\text { Ionic } \\
\text { Cooling }
\end{array}\right) \\
q(0) & ={ }^{q_{I B}}+\sum_{i} q_{i}+\sum_{j} \frac{3 / 2(N k T)}{T_{j}}+\frac{3 / 2(N k T)}{T}
\end{aligned}
$$

where $q_{I B}=e \cdot v \cdot /(g-s e c \cdot)$ that appears as inner bremsstrahlung $\mathrm{X}$-radiation,

$q_{i}=e_{\cdot} \cdot /\left(g-s_{0}\right)$ that eventually emerges as the spectral line $\left(h \nu_{i}\right)$,

$T_{j}=$ relaxation time (sec.) for cooling by the ( $j$ ) th atomic species, $\tau_{+}=$relaxation time (sec.) for cooling by collisional excitation of several ionic species via intermediary electrons,

$\mathrm{kT}=10^{-2} \mathrm{e} \cdot \mathrm{v} \cdot\left(\right.$ for $\mathrm{T} \approx 10^{2} \mathrm{~K}$ ),

$\mathrm{N}$ = Avagadro Number $\left(6 \times 10^{23}\right)$. 
The $\mathrm{X}$-radiation from inner bremsstrahlung is the only output of the cloud that directly tracks the spectrum of the cosmic ray nucleons, but as far as the energy balance (2) is concerned the $\mathrm{X}$-rays make an entirely negligible contribution. As described by Hayakawa, Nishimura, and Takayanagi (1961), the important hydrogen atomic emission lines in this situation are Ly- $\alpha, \mathrm{H} \alpha$, and $\mathrm{P} \alpha$. Smith (1966) has estimated that near $\mathrm{T} \sim 10^{2}{ }^{\circ} \mathrm{K}$, the major contribution to cooling by atomic collisional excitation is accounted for by the interstellar abundance of oxygen. The relaxation time $\left(\tau_{0}\right)$ for this process is not a sensitive function of temperature in the vicinity of $10^{2}{ }^{\circ} \mathrm{K}$ and is given by

$$
\tau_{0}=12 \times 10^{11} \mathrm{sec} \rightarrow 4 \times 10^{4} \text { years. }
$$

For the case where the electron concentration is high $\left(\mathrm{n}_{\mathrm{e}} \geq 10^{-1} \mathrm{~cm}^{-3}\right)$, cooling by the Seaton (1955) mechanism of ionic collisional excitation by electrons becomes important. To provide for this we use a value of $\tau_{+}$extracted from the work of Takayanagi and Nishimura (1960), viz

$$
\tau_{+}=\frac{10^{11}}{n_{e}} \mathrm{sec} .
$$

The ion-electron density $\left(\mathrm{n}_{\mathrm{e}}\right)$ produced in $\operatorname{cool}\left(\sim 10^{2}{ }^{\circ} \mathrm{K}\right)$ interstellar $\mathrm{H} 1$ regions (Hayakawa, 1960) by cosmic rays is fixed by $q(0)$. For a steady-state situation in a medium of pure hydrogen 
the production via the ionization process is balanced by recombination as follows:

$$
\sigma_{\mathrm{r}} \mathrm{v}_{\mathrm{e}} \mathrm{n}_{\mathrm{e}}^{2}=\left(\frac{\mathrm{q}}{\Delta \mathrm{E}}\right) \cdot\left(\frac{\mathrm{n}_{\mathrm{H}}}{\mathrm{N}}\right)
$$

where: $\sigma_{\mathrm{r}}=2 \times 10^{-19} \mathrm{~cm}^{2}=$ recombination cross section (Bethe and Salpeter, 1957) at $\mathrm{T}=10^{2}{ }^{\circ} \mathrm{K}$, $\mathrm{V}_{\mathrm{e}}=$ thermal electron velocity at $10^{2}{ }^{\circ} \mathrm{K}$, $\mathrm{n}_{\mathrm{H}}=\mathrm{H} 1$ hydrogen number density $\left(\sim 10 \mathrm{~cm}^{-3}\right)$, $\Delta \mathrm{E}=36 \mathrm{e} \cdot \mathrm{v} \cdot=$ mean energy dissipated by a cosmic ray per ion pair formed (Rossi, 1952), $\mathrm{N}=$ Avagadro Number

From (5) we obtain at $10^{2}{ }^{\circ} \mathrm{K}$ that

$$
\mathrm{n}_{\mathrm{e}}=7 \times 10^{-7} \mathrm{q}(\rho) \mathrm{cm}^{1 / 2}
$$

The insertion of this expression (6) for $\mathrm{n}_{e}$ into (4) gives $\tau_{+}$ directly in terms of $q(0)$, viz

$$
\tau_{+}=\frac{10^{18}}{7} \quad \mathrm{q}(0)^{-1 / 2} \mathrm{sec} .
$$

From the work of Hayakawa (1963) and Hayakawa, Nishimura and Takayanagi (1961), we estimate

$$
\mathrm{q}_{\mathrm{IB}}=3 \times 10^{-5} \quad \mathrm{q}(0)
$$




$$
\sum q_{i}=4 \times 10^{-1} q(0)
$$

The substitution of (3), (7), (8), and (9) into (2) yields an equation for $q(0)$ alone, with the solution

$$
q(o)=3 \times 10^{10} \mathrm{e} \cdot \mathrm{v} \cdot /(\mathrm{g}-\mathrm{sec})
$$

This thermal solution (10) for $q(0)$ may be used to obtain the corresponding thermal solution for $n_{e}$, via (6), as

$$
\mathrm{n}_{\mathrm{e}} \approx 10^{-1} \mathrm{~cm}^{-3}
$$

This value for $\mathrm{n}_{e}$ is likely to be an upper limit since there might be other recombination processes competitive to simple electron-proton attachment. On the basis of the low frequency $\left(<5 \times 10^{6} / \mathrm{sec}\right)$ fall-off for the galactic radio spectrum in directions towards the galactic poles, Hoyle and Ellis (1963) conclude that $\left\langle n_{e}\right\rangle$, the average electron density in our region of the galactic disk, is given by

$$
\left\langle n_{e}\right\rangle \approx 10^{-4} \mathrm{~T}^{3 / 4}
$$

If the electrons responsible for the low frequency galactic radio absorption by the galactic disk reside mainly in $\mathrm{H} 1$ clouds then $\mathrm{T} \approx 10^{2}{ }^{\circ} \mathrm{K}$ for these electrons. That $\mathrm{H} 1$ clouds occupy $\sim 10 \%$ of 
the volume of the galactic disk would imply that $\mathrm{n}_{\mathrm{e}} \approx 10\left\langle\mathrm{n}_{\mathrm{e}}\right\rangle$; whereby (12) gives

$$
\mathrm{n}_{\mathrm{e}} \approx 10\left\langle\mathrm{n}_{\mathrm{e}}\right\rangle \approx 10^{-1} \mathrm{~cm}^{-3}
$$

Radiation

The electromagnetic radiation from $\mathrm{Hl}$ clouds is a measurable consequence of the cosmic ray phenomenon described here. The intensity $\left(I_{1}\right)$ of various spectral lines is given by

$$
I_{i}=\left(\frac{s q_{i}}{4 \pi N}\right) \frac{e}{c m} \frac{v}{s e c-s r}
$$

where (S) is the optical thickness in hydrogen atoms $/ \mathrm{cm}^{2}$ for penetrating radiation (e.g. $\mathrm{H} \alpha$ ); for diffuse radiation (e.g. Ly- $\alpha$ )

(S) is comparable to the thickness of the galactic disk, in hydrogen atoms $/ \mathrm{cm}^{2}$.

Using the results of Hayakawa, Nishimura and Takayanagi (1961)

for $q_{i} /\left(\sum q_{i}\right)$ with the approximation (9), $q(0)$ given by $(10), s=10^{21} / \mathrm{cm}^{2}$ for $L y-\alpha$, and $S=10^{22}$ for $H_{\alpha}$, we obtain

$$
\begin{aligned}
& \mathrm{I}_{\mathrm{Ly} \alpha} \simeq 10^{6} \mathrm{e} \cdot \mathrm{v} \cdot /\left(\mathrm{cm}^{2}-\mathrm{sec}-\mathrm{sr}\right) . \\
& \text { and } \\
& \mathrm{I}_{\mathrm{H} \alpha} \simeq 10^{6} \mathrm{e} \cdot \mathrm{v} \cdot /\left(\mathrm{cm}^{2}-\mathrm{sec}-\mathrm{sr}\right) .
\end{aligned}
$$


This value (16) for galactic $\mathrm{H} \alpha$ radiation corresponds to $\sim 2$ Rayleighs, which is within an order of magnitude of the Ho intensity associated with the galactic disk, $(\sim 5 \rightarrow 20$ Rayleighs $)$ as measured by Dufay, Dufay and Huu-Doan (1961).

From the work of Hayakawa (1963) we may estimate that, for inner bremsstrahlung,

$\frac{d I}{d h \nu} \simeq 3 \times 10^{-9} \frac{(s)}{4 \pi} q\left(E_{0}=500(h v)\right)_{\left(\overline{c m} 2-\frac{e \cdot v \cdot}{s e c-s r-e \cdot v \cdot)}\right.} \cdot$

Substitution of the definition (1) for $q$ into (17) yields an integral transform for the interstellar cosmic ray proton spectrum $(\mathrm{dJ} / \mathrm{dE})$, viz

$$
\left(\frac{\mathrm{dI}}{\mathrm{dh \nu}}\right)_{h \nu} \approx\left[\left(3 \times 10^{-9}\right) \mathrm{s} \int_{(500 \mathrm{~h} \nu)}^{\infty}\left(\frac{\mathrm{dJ}}{\mathrm{dE}}\right)\left(\frac{\mathrm{dE}}{\mathrm{dx}}\right) \frac{\mathrm{dE}}{r}\right] \frac{\left.\mathrm{e} \cdot \mathrm{v} \cdot \mathrm{cm}^{2}-\mathrm{sec}-\mathrm{sr}-\mathrm{e} \cdot \mathrm{v} \cdot\right)}{(18)}
$$

Since the ionization loss rate $(\mathrm{dE} / \mathrm{dx})$ is known as a function of $\mathrm{E}$, a determination of $(\mathrm{dI} / \mathrm{dh} \nu)$ for the inner bremsstrahlung $\mathrm{X}$-rays gives a direct representation of the cosmic ray proton spectrum.

Consider the $\mathrm{X}$-ray spectral intensity at hv $\approx 30 \mathrm{keV}$; this corresponds to $\mathrm{EO}_{\mathrm{O}}=15 \mathrm{MeV} /$ nucleon for the interstellar cosmic ray spectrum. As described in the next section (see Table 1), a consistent interstellar spectrum gives $\mathrm{q}($ Eo $) \approx 3 \times 10^{10} \mathrm{e} \cdot \mathrm{v} \cdot /(\mathrm{g}-\mathrm{sec})$ 
for Eo $=15 \mathrm{MeV} /$ nucleon. The spectral intensity of $30 \mathrm{keV}$ $\mathrm{X}$-rays for directions that traverse most of the galactic disk is obtained by inserting $\mathrm{S} \approx 10^{23}$ atoms $/ \mathrm{cm}^{2}$ into (17) and is evaluated as

$$
\left(\frac{\mathrm{dI}}{\mathrm{dh \nu}}\right)_{30 \mathrm{keV}} \approx 1 \quad \frac{\mathrm{e} \cdot \mathrm{v} \cdot}{\left(\mathrm{cm}^{2}-\mathrm{sec}-\mathrm{sr}-\mathrm{e} \cdot \mathrm{v} \cdot\right)}
$$

This intensity (19) is comparable to the observed background (Bleeker et al., 1966) of X-rays at $\sim 30 \mathrm{keV}$. It is important to note that a marked anisotropy is expected for the X-rays discussed here since the intensity viewed normal to the galactic plane would be diminished by an order of magnitude. Measurements of the nonstellar X-rays from HI clouds should yield the spatial distribution and spectrum of the low energy $\left(<10^{2} \mathrm{MeV} /\right.$ nucleon $)$ cosmic rays that propagate throughout the galactic disk.

Interstellar Cosmic Ray Spectrum

Recent measurements (Balasubrahmanyan et a1., 1966; Comstock et al., 1966; Fan et a1., 1966; Biswas et al., 1966; Meyer et a1., 1967; Hormann and Winckler, 1966; Ormes and Webber, 1966) of the spectrum for cosmic ray $\mathrm{H}^{1}, \mathrm{H}^{2}, \mathrm{H}^{3}, \mathrm{He}^{3}, \mathrm{He}^{4}, \mathrm{C}^{12}, \mathrm{~N}^{14}$ and $\mathrm{O}^{16}$ nuclei indicate that: 
i) the interstellar spectrum for cosmic ray alpha particles is similar in form to that for protons, on a kinetic energy per nucleon basis (Balasubrahmanyan et al., 1967 ), over the band of maximum observed flux (i.e. $10^{8} \rightarrow 10^{9}$ e.v./nucleon),

ii) the mean cosmic ray traversal through the interstellar medium is $\sim 6 \mathrm{~g} / \mathrm{cm}^{2}$, but could be as low as $3 \mathrm{~g} / \mathrm{cm}^{2}$ at the upper and lower energy extremes of the spectrum (Fan et al., 1966; Biswas et a1., 1966; Meyer et al., $1967)$,

iii) the spectral shape for $\mathrm{C}, \mathrm{N}$, and $\mathrm{O}$ nuclei (Comstock et al., 1966) relative to alpha particles at low energies (< $500 \mathrm{MeV} / \mathrm{nucleon}$ ) may be explained (Cowsik et al., 1966) by a steady-state model whereby the interstellar path distribution is exponential, favoring short paths, and

iv) the source spectrum for cosmic ray protons and alpha particles cannot flatten at low energies, as would be the case for a power law in total energy (Meyer et al., $1967)$

A proton source spectrum that is compatible with present observations is :

$$
\left(\frac{\mathrm{dJ}}{\mathrm{dE}}\right)_{0}=\frac{\left(\mathrm{E} / \mathrm{m}_{\mathrm{o}} \mathrm{c}^{2}\right)^{-2.5}}{\mathrm{~m}_{\mathrm{o}} \mathrm{c}^{2}} \quad\left(\text { unit E-cm }{ }^{2}-\mathrm{sr}-\mathrm{sec}\right)^{-1}
$$


where $E$ is the kinetic energy and $m_{0} c^{2}$ is the rest energy.

This source spectrum (20) is to be modified by the energy degradation of the protons that traverse an exponential distribution (Cowsik et al., 1966) of interstellar paths (X), the calculation has been made for both $\langle x\rangle=6 \mathrm{~g} / \mathrm{cm}^{2}$ and $\langle x\rangle=3 \mathrm{~g} / \mathrm{cm}^{2}$. These spectra are exhibited in Figure 1, along with a curve given by Gloeckler and Jokipii (1967) as a summary of proton measurements made during 1965, a period of minimum solar modulation. The ratio of the interstellar spectrum $(\mathrm{dJ} / \mathrm{dE})_{\mathrm{x}}$ for $\langle\mathrm{X}\rangle=6 \mathrm{~g} / \mathrm{cm}^{2}$ to the spectrum observed in 1965 is plotted as a function of $1 / \beta$ in Figure 2. Over the band $50 \rightarrow 800 \mathrm{MeV} /$ nucleon, where solar modulation is significant, we note that the ratio may be approximated by

$$
\frac{(d J / d E)_{x}}{(d J / d E)_{1965}}=\exp (2.7 / \beta)
$$

This formula (21) for the solar modulation of cosmic rays over the indicated energy band is of the form shown by Balasubrahmanyan, Boldt and Palmeira (1967) to be an adequate description during several years near the period of minimum solar activity.

The interstellar cosmic ray proton spectrum discussed here $\left((\mathrm{dJ} / \mathrm{dE})_{\mathrm{X}}\right)$, exhibited in Figure 1 , may be used to estimate a value for q consistent with cosmic ray particle observations. 
The contributions to $\mathrm{q}$ from the energy losses by other nuclei (mainly $\mathrm{He}^{4}$ ) is here approximated (Balasubrahmanyan et al., 1967) by taking $n \simeq 1 / 2$ in equation (1). Using the known energy degradation of protons traversing atomic hydrogen for energies above $E_{0}$ (15 MeV) we obtain from (1) that $q$ for the interstellar spectrum proposed here (see Fig. 1) is:

$$
\begin{aligned}
& q\left(E_{o}\right)=(2-4) \times 10^{10} \mathrm{e} \cdot \mathrm{v} \cdot / \mathrm{g}-\mathrm{sec} \\
& \text { for }\langle\mathrm{x}\rangle=(3-6) \mathrm{g} / \mathrm{cm}^{2}
\end{aligned}
$$

Shifting $E_{o}$ by $10 \mathrm{MeV} /$ nucleon changes this value of $q$ by less than $50 \%$. As shown in Table 1, the interstellar spectrum discussed by Gloeckler and Jokipii (1967) gives a comparable value of $q$ for $E_{0}$ $=15 \mathrm{MeV}$. However, the latter spectrum steepens sharply below $50 \mathrm{Mev} /$ nucleon and this has the effect of changing $q$ by over an order of magnitude when $E_{O}$ is lowered by $\leq 10 \mathrm{MeV}$.

The value of $q$ obtained (22) on the basis of this spectral analysis of observed cosmic rays matches the value (10) for the thermal solution. Therefore, it appears consistent to attribute the heating of $\mathrm{H} 1$ regions mainly to the Coulomb interactions of cosmic rays. Models of the interstellar spectrum are compared for this role in Table 1; a previous extreme estimate based on a minimal solar modulation (Balasubrahmanyan et al., 1966; Brunstein and Cline, 1966) is seen to be inadequate (see Table 1, last entry). 
A measure of the electromagnetic energy deposition by cosmic rays during a lifetime is given by

$$
\mathrm{q}\left\langle\frac{\mathrm{x}}{\mathrm{v}}\right\rangle \simeq \mathrm{q} \frac{<\mathrm{x}\rangle}{\mathrm{c}} \quad\left(\mathrm{e} \cdot \mathrm{v} \cdot / \mathrm{cm}^{3}\right)
$$

As exhibited in Table 1, this is comparable to the kinetic energy density for the interstellar proton spectrum examined here (Fig. 1). Most of this kinetic energy density is accounted for by those protons of high ionizing power; the kinetic energy density for the relativistic protons (E $>500 \mathrm{MeV}$ ) of this interstellar spectrum is less than $1 \mathrm{e} \cdot \mathrm{v} \cdot / \mathrm{cm}^{3}$. The power $\mathrm{U}$ dissipated by all cosmic rays via Coulomb interactions with the entire mass (Ginzburg and Syrovatskii, 1964) of galactic gas $\left(\sim 3 \times 10^{42} \mathrm{~g}\right)$ is given by:

$$
\begin{aligned}
& \mathrm{U}=\mathrm{Mq}=\left(3 \times 10^{42} \mathrm{~g}\right)\left(2 \times 10^{10} \mathrm{e} \cdot \mathrm{v} \cdot /(\mathrm{g}-\mathrm{sec})\right) \\
& =6 \times 10^{52} \mathrm{e} \cdot \mathrm{v} \cdot / \mathrm{sec} \text {. } \\
& \rightarrow 4 \times 10^{40} \mathrm{ergs} / \mathrm{sec} \text {. }
\end{aligned}
$$

This value of $U$ for the dissipated power is equal to the total power discussed by Ginzburg and Syrovatskii (1964) as required for the steady-state replenishment of cosmic rays that escape from the galaxy.

\section{Acknowledgements}

It is a pleasure to acknowledge valuable discussions with A. Klimas, F. B. McDonald, and J. P. Meyer, and to thank J. P. Meyer 
for making his calculations on interstellar traversal available to us prior to publication.

This report summarizes a paper delivered by E. Boldt at the Tenth International Conference on Cosmic Rays, Calgary, Canada, June 29, 1967. An elaboration of the subject of this paper is being prepared for inclusion in a volume of contributions dedicated to Bruno Rossi, edited by G. Sandri. 


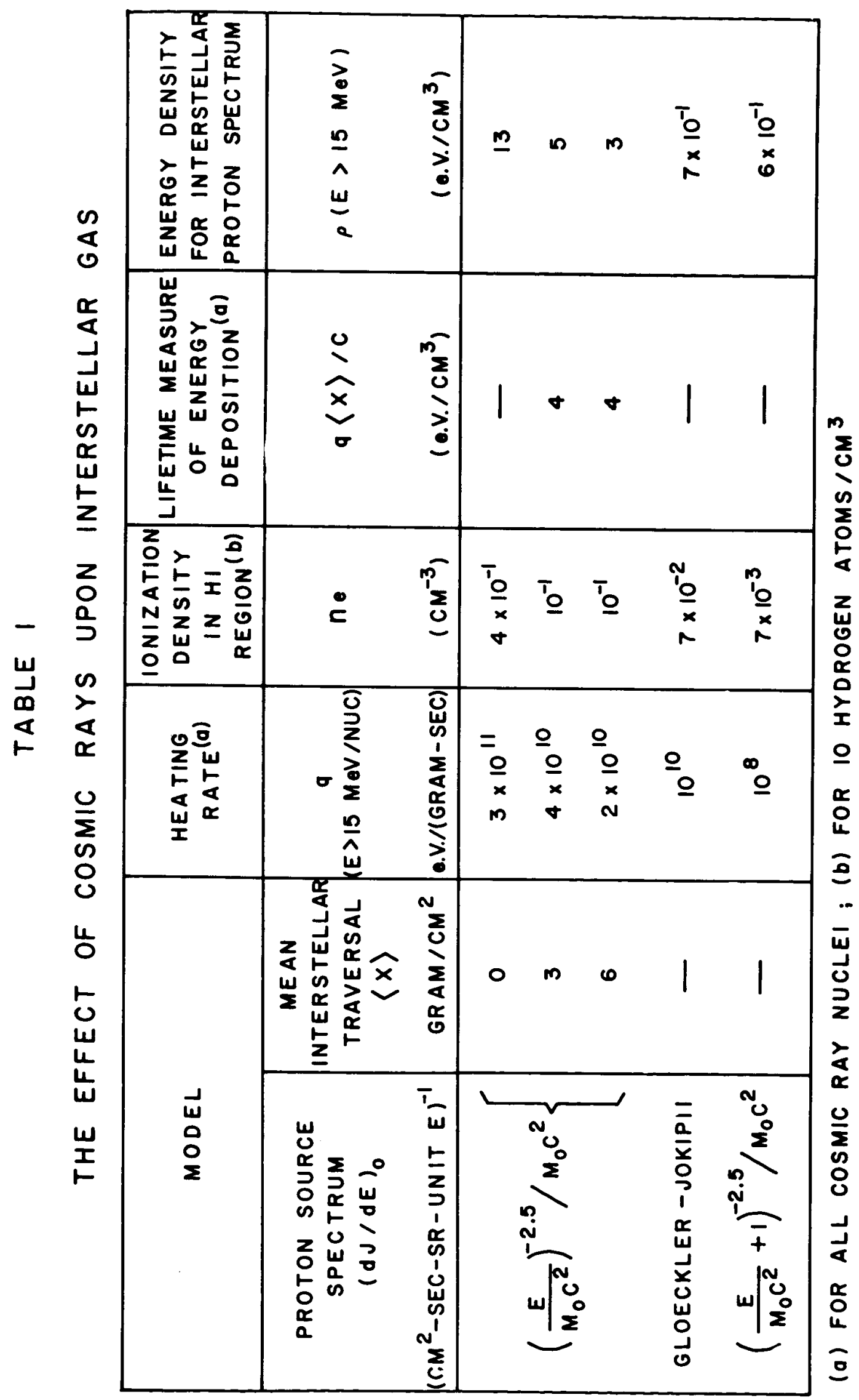


Balasubrahmanyan, V. K., Hagge, D. E., Ludwig, G. H., and McDonald, F. B., Proc. Ninth Internat. Conf. Cosmic Rays (London) (London: Institute of Physics and Physical Society 1, 427 (1966) and J. Geophys. Res., 71, 1771 (1966).

Balasubrahmanyan, V. K., Boldt, E., and Palmeira, R. A. R., J. Geophys. Res. 72,27 (1967).

Balasubrahmanyan, V. K., Boldt, E., and Palmeira, R. A. R., Phys. Rev. 140, B 1157 (1965).

Bethe, H. A. and Salpeter, E. E., Quantum Mechanics of One- and TwoElectron Atoms (1957), Academic Press, Inc. (New York).

Biswas, S., Ramadurai, S., and Sreenivasan, N., Tata Institute of Fundamental Research N.E. 66-19 (1966) and Phys. Rev. 149, 1038 (1966)

Bleeker, J. A. M., Burger, J. J., Scheepmaker, A., Swanenburg, B. N., and Tanaka, Y., Phys. Letters 21,301 (1966).

Brunstein, K. A. and Cline, T. L., Nature 209, 1186 (1966).

Comstock, G. M., Fan, C. Y., and Simpson, J. A., Ap. J. 146, 51 (1966). Cowsik, R., Yash Pal, Tandon, S. N., and Verma, R. P., Tata Institute of Fundamental Research N.E. 66-18 (1966)

Dieter, N. H. and Goss, W. M., Rev. Mod. Phys. 38, 256 (1966)

Dufay, J., Dufay, M., and Huu-Doan, N., Compt. Rend. Acad. Sci., Paris $253,974(1961)$.

Fan, C. Y. Gloeckler, G., and Simpson, J.A., Phys. Rev. Letters 17, 329 (1966). 
Field, G. B., The Distribution and Motion of Interstellar Matter in Galaxies (Ed. L. Woltjer), 1962, Benjamin (New York).

Ginzburg, V. L. and Syrovatskii, S. I., The Origin of Cosmic Rays (1964) Pergamon Press (Oxford).

Gloeckler, G. and Jokipii, J. R., Ap. J. 148, L41 (1967).

Hayakawa, S., Pub1. A. S. Japan 12, 110 (1960).

Hayakawa, S., Nishimura, S., and Takayanagi, K., Publ. A. S. Japan 13, $184(1961)$.

Hayakawa, S., Brandeis Summer Institute in Theoretical Physics Vol. 2 (1963) Brandeis University (1964).

Hofman, D. J. and Winckler, J. R., Phys. Rev. Letters 16, 109 (1966).

Hoyle, F. and Ellis, G. R. A., Aust. J. Phys. 16, 1 (1963).

Kahn, F. D., Gas Dynamics of Cosmic Clouds, ed. van de Hulst and Burgers (North-Holland Publ. Co. 1955) P. 60

Meyer, J. P., Hagge, D. E., and McDonald, F. B., post deadline paper at the Washington, D. C. meeting of the American Physical Society, April 26, 1967 and presented at Tenth Internat. Conf. Cosmic Rays, Calgary, Canada, June 1967.

Ormes, J. F. and Webber, W. R., Proc. Ninth Internat. Conf. Cosmic Rays (London) (London: Institute of Physics and Physical Society) 1, 349 (1966).

Rossi, B., High Energy Particles, 1952, Prentice-Ha11, Inc. (New York). Seaton, M. J., Annls. Astrophys. 18, 188 (1955).

Smith, F. J., Planet. Space Sci. 14, 937 (1966).

Takayanagi, K. and Nishimura, S., Publ. A. S. Japan 12, 77 (1960). 


\section{Figure Captions}

Figure 1. The "observed" curve is a summary of the proton spectrum as given by Gloeckler and Jokipii (1967), based upon balloon and satellite borne observations by several laboratories (Balasubrahmanyan et a1., 1966; Comstock et a1., 1966; Fan et a1., 1966; Biswas et a1., 1966; Meyer et al., 1967; Hofman and Winckler, 1966; Ormes and Webber, 1966) during 1965. The curves " $\left\langle 3 \mathrm{~g} / \mathrm{cm}^{2}>"\right.$ and " $<6 \mathrm{~g} / \mathrm{cm}^{2}>"$ represent the indicated power law source spectrum after traversing interstellar matter via an exponential distribution of paths with a mean of $3 \mathrm{~g} / \mathrm{cm}^{2}$ and $6 \mathrm{~g} / \mathrm{cm}^{2}$ respectively.

Figure 2. The dotted curve is the ratio of the interstellar spectrum for $\left\langle 6 \mathrm{~g} / \mathrm{cm}^{2}\right\rangle$ as shown in Fig. 1 to the spectrum observed in 1965 (Fig. 1). The solid line is $\exp (2.7 / B) . \quad \beta$ is the proton velocity in units of the velocity of light (c). 


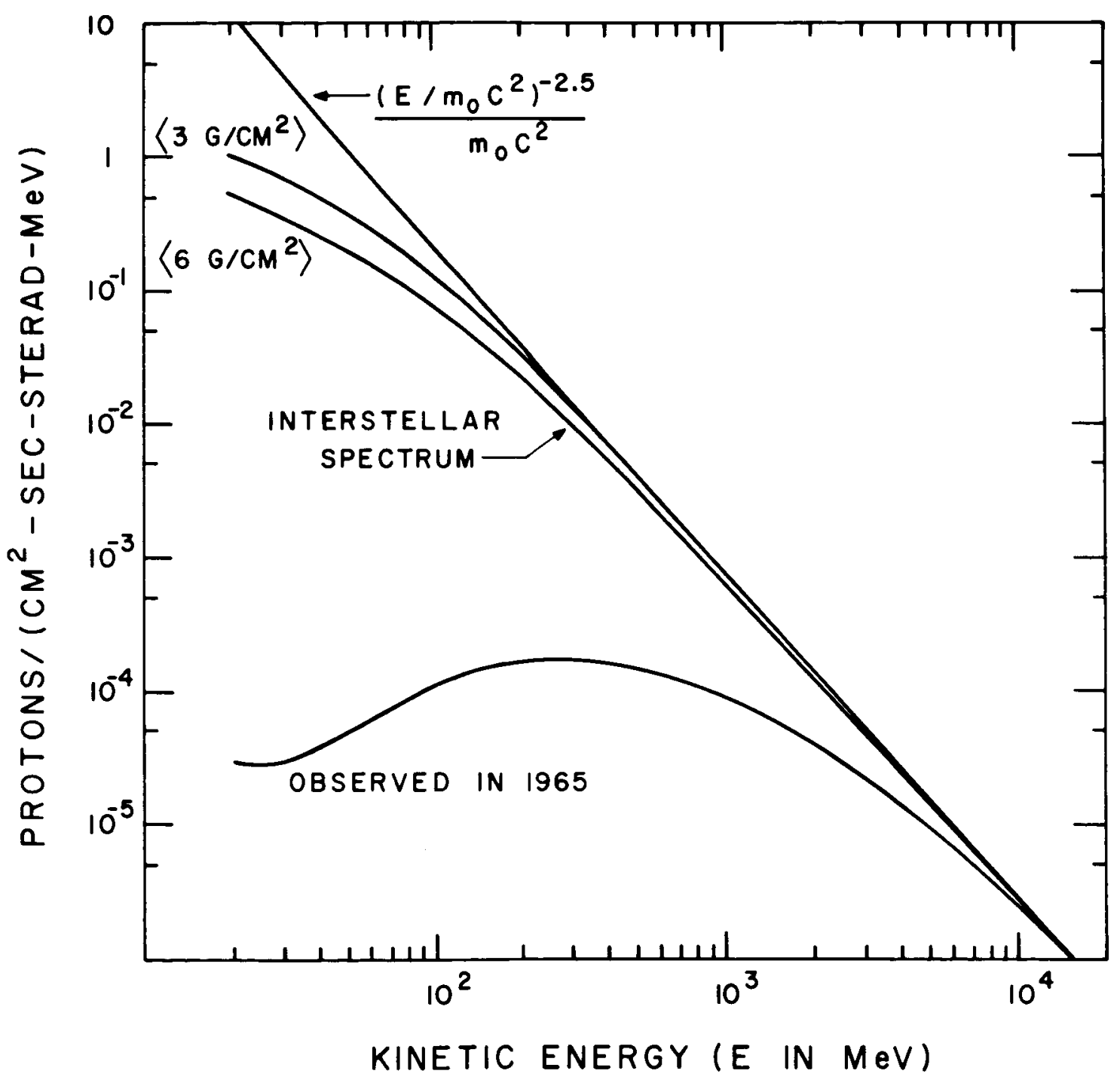




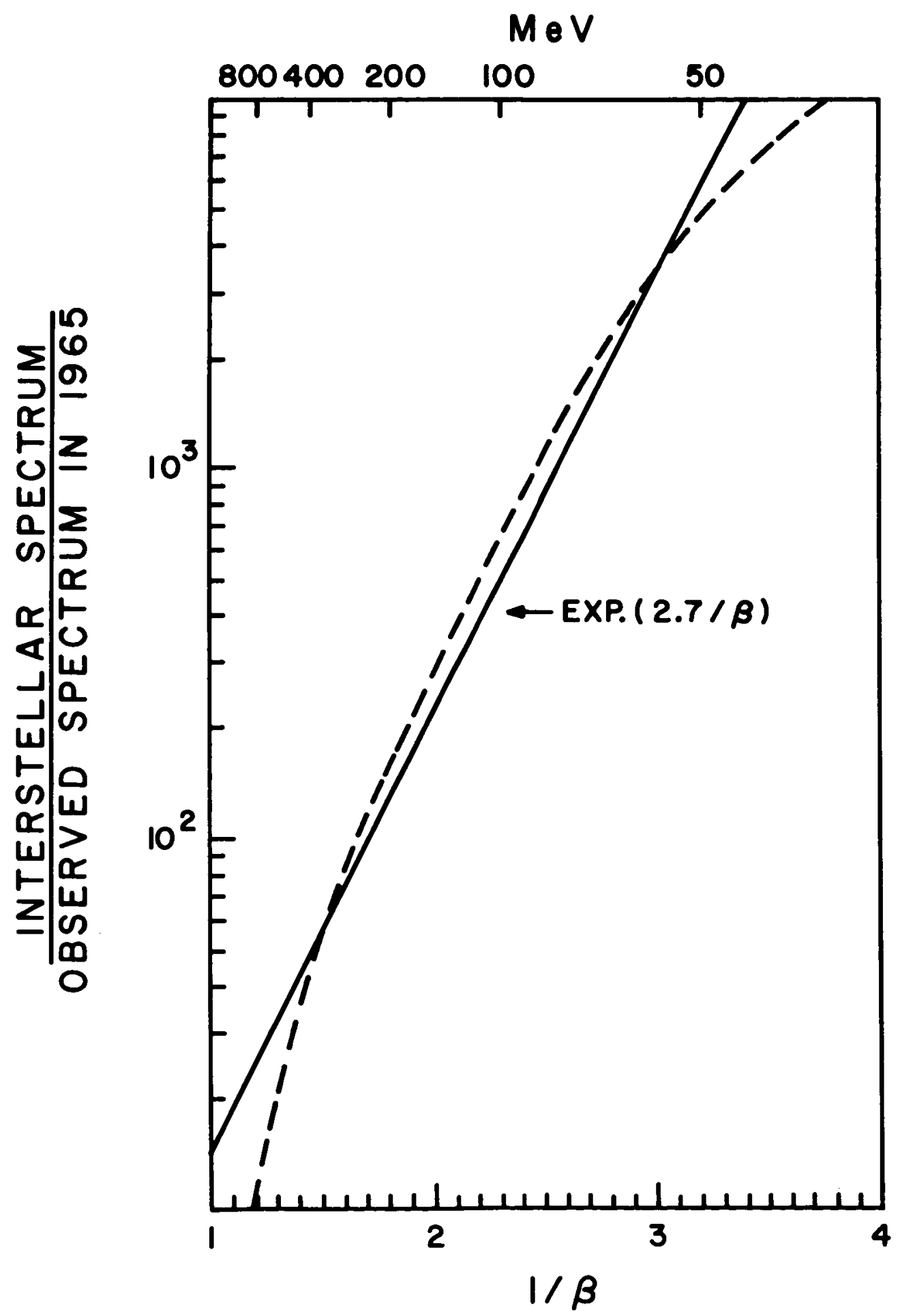

